\title{
PortuCol: uma pseudolinguagem inspirada em C ANSI para o Ensino de Lógica de Programação e Algoritmos
}

\author{
Lucas Lemos Barbosa, Christian Marlon Souza Couto, Ricardo Terra \\ Departamento de Ciência da Computação, \\ Universidade Federal de Lavras (UFLA), Brasil \\ lbarbosalcomputacao.ufla.br, christianmscesistemas.ufla.br, terraddcc.ufla.br
}

\begin{abstract}
Resumo. Linguagens de programação são intrinsecamente complexas, devido à variabilidade, generalidade e completude de suas construções. Isso justifica a adoção de pseudolinguagens de alto nível no ensino de Lógica de Programação e Algoritmos. Embora C ANSI e Java sejam as linguagens de programação mais utilizadas, Portugol-uma pseudolinguagem com instruções em português inspirada na linguagem obsoleta Pascal - é ainda largamente adotada por IES. Diante desse cenário, este artigo propõe PortuCol, uma pseudolinguagem de programação com instruções em português assim como Portugol, porém inspirada em C ANSI. Este artigo também descreve PortuCol2C, uma ferramenta que traduz código escrito em PortuCol para C ANSI e conduz estudos empíricos que demonstram estatisticamente que (i) PortuCol é $27 \%$ mais similar a C ANSI do que o Portugol e (ii) PortuCol é para C ANSI o que Portugol é para Pascal.
\end{abstract}

\begin{abstract}
Programming languages are intrinsically complex due to the variability, generality, and completeness of their constructions. This justifies the adoption of high-level pseudocode languages when teaching Introduction to Algorithms. Although ANSI C and Java are the most used programming languages, Portugol-a pseudocode language with instructions in Portuguese inspired by the outdated Pascal language - is still widely adopted by Brazilian universities. In view of such circumstances, this paper proposes PortuCol, a pseudocode programming language with instructions in Portuguese as Portugol, although inspired by ANSI C. It also describes PortuCol2C, a tool that translates PortuCol to ANSI C, and conducts empirical studies that statistically demonstrate that (i) PortuCol is 27\% more similar to ANSI C than Portugol and (ii) PortuCol is for C ANSI what Portugol is for Pascal.
\end{abstract}

\section{Introdução}

No início do ensino superior, é comum que estudantes da área de Ciência da Computação e afins tenham dificuldade no raciocínio lógico de criação de soluções, uma vez que, no ensino médio, esses estudantes estavam acostumados a apenas aplicar fórmulas previamente existentes na solução de problemas. Um estudo atesta essa dificuldade reportando que, em média, mais de $50 \%$ dos alunos das disciplinas de introdução à programação são reprovados a cada semestre [2].

Em virtude de que linguagens de programação são intrinsecamente complexas devido à variabilidade, generalidade e completude de suas construções - docentes de IES 
usualmente adotam pseudolinguagens de alto nível nas disciplinas de introdução à Lógica de Programação e Algoritmos. Pseudolinguagens proporcionam ao aluno um maior espaço para o desenvolvimento de seu raciocínio lógico relacionado à resolução de problemas, sem preocupações relativas ao uso correto de uma linguagem de programação.

Portugol é, hoje no Brasil, a pseudolinguagem mais adotada por universidades com esse propósito [9, 11], a qual conta com diversas ferramentas de apoio ao ensino $[3,5,9,8,4]$. No entanto, Portugol é o produto de uma simbiose do português e as linguagens de programação ALGOL e Pascal [5], ambas obsoletas [10]. Pascal, por exemplo, sequer está classificada entre as 50 linguagens de programação mais utilizadas no desenvolvimento de sistemas de software [10]. Assim, devido à origem sintática de Portugol, estudantes não terão uma transição natural ao aprender linguagens de programação do estado-da-prática como C ANSI e Java.

Como uma alternativa ao Portugol, este artigo propõe PortuCol, uma pseudolinguagem de programação com instruções em português assim como Portugol, porém inspirada em C ANSI. Estudos empíricos atestam que (i) PortuCol é 27\% mais similar a C ANSI do que o Portugol, o que proporciona uma transição mais natural para o estudante ao aprender linguagens do estado-da-prática e (ii) PortuCol é para C ANSI o que Portugol é para Pascal, o que retrata apenas uma adaptação da pseudolinguagem às linguagens do estado-da-prática. Além disso, este artigo descreve PortuCo12C, uma ferramenta que traduz código PortuCol para C ANSI, o que possibilita aos estudantes se familiarizem com a linguagem C ANSI através da observação do seu código PortuCol traduzido.

Este artigo está organizado como a seguir. A Seção 2 apresenta Portugol, a pseudolinguagem mais adotada em disciplinas de programação do Brasil. A Seção 3 descreve o PortuCol, sua sintaxe e a ferramenta PortuCol2C. A Seção 4 reporta os resultados da avaliação de similaridade textual entre algoritmos implementados em PortuCol, C ANSI, Portugol e Pascal. Por fim, a Seção 5 conclui e propõe trabalhos futuros.

\section{Portugol}

O Portugol é a pseudolinguagem mais adotada em classes introdutórias à programação em IES no Brasil [9, 11]. Mais importante, a maioria dos livros utilizados como referência básica nas disciplinas de introdução à programação em universidades brasileiras possui explicações usando alguma notação de Portugol [9, 11]. Em suma, o objetivo de Portugol é facilitar o aprendizado de Lógica de Programação e Algoritmos para alunos não habituados com programação.

Portugol é o produto de uma simbiose do português e as linguagens de programação ALGOL e Pascal [5], ambas obsoletas [10]. Seu propósito - o qual foi atingido é ser uma pseudolinguagem simples e com instruções em português para favorecer estudantes não familiares com a língua inglesa. Ao utilizar Portugol, o aluno não precisa se preocupar com a sintaxe e comandos em inglês que uma linguagem de programação mais complexa possui, o que foca sua concentração no como resolver um problema proposto.

Apesar de trazer benefícios inquestionáveis no aprendizado de Lógica de Programação e Algoritmos, o Portugol não provê, por definição, uma forma de executar o pseudocódigo escrito para que alunos possam testar seus algoritmos, o que se torna indispensável à medida que os algoritmos se tornam mais complexos e passam a requerer códigos 
mais elaborados. Esse problema ocorre pois o Portugol tem diversas variações em sintaxe e em comandos dependendo do contexto de utilização. Isso faz com que as ferramentas não sejam padronizadas, i.e., suportando sintaxes e instruções diferentes $[3,5,9,8,4]$.

Dentre as ferramentas existentes que apoiam o ensino utilizando Portugol podemos destacar: CIFluxProg [3] disponibiliza um ambiente visual com fluxogramas, além de um ambiente textual com uma variação de Portugol; WEB-UNERJOL [5] provê um ambiente web que permite o desenvolvimento e execução de algoritmos em Portugol através de um navegador; Portugol Studio [9] é um ambiente de desenvolvimento integrado (IDE) para uma variante do Portugol que utiliza o compilador Portugol Núcleo; Portugol IDE [8] provê um ambiente que possibilita desenvolver algoritmos em Portugol através do uso de fluxogramas como uma linguagem gráfica, além do tradicional modo textual; e, por fim, VisuAlg[4] que adota uma variante de Portugol bem parecida com Pascal, porém sem pontos-e-vírgulas para separar comandos. Os Algoritmos 1 e 2 ilustram tal similaridade apresentando um mesmo algoritmo escrito em Portugol e em Pascal, respectivamente.

Algoritmo 1. Portugol

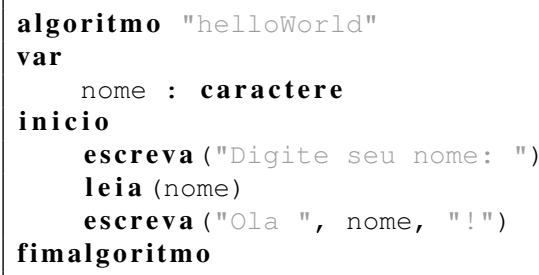

Algoritmo 2. Pascal

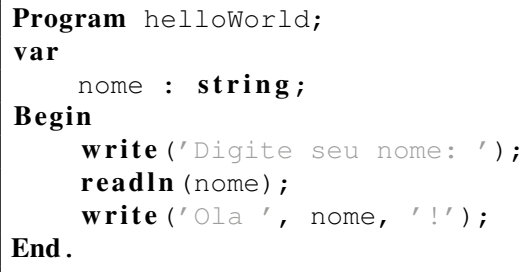

\section{PortuCol}

Este artigo propõe PortuCol, uma pseudolinguagem de programação com instruções em português assim como Portugol, porém inspirada em C ANSI. O objetivo é adaptar o ensino de Lógica de Programação e Algoritmos através de uma pseudolinguagem cuja sintaxe seja inspirada em uma linguagem de programação do estado-da-prática (i.e., amplamente adotada por fábricas de software).

A Figura 1 ilustra o contexto de utilização de PortuCol. Estudantes desenvolvem o código em PortuCol e, automaticamente, a ferramenta PortuCol2C traduz PortuCol para C ANSI e executa o código correspondente. Isso possibilita que estudantes (i) executem seu pseudocódigo; (ii) se familiarizem com a linguagem C ANSI através da observação do seu código PortuCol traduzido; e (iii) tenham uma transição mais natural ao futuramente aprender linguagens do estado-da-prática como C ANSI e Java.

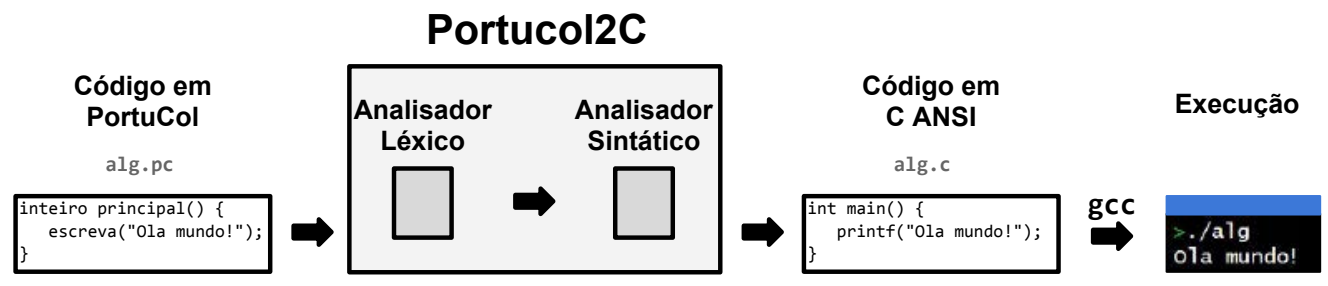

Figura 1. Utilizando PortuCol 


\subsection{Sintaxe}

A definição da sintaxe de PortuCol considerou didática, facilidade de aprendizagem e similaridade com C ANSI de forma que futuramente a transição para essa linguagem de programação seja mais natural para o aluno.

A Tabela 1 apresenta a equivalência de instruções PortuCol, C e Portugol. PortuCol possui instruções e palavras reservadas em português similarmente à Portugol e uma sintaxe similar à C ANSI. Em PortuCol, assim como em C ANSI, é necessário delimitar o escopo do código com os símbolos de abre e fecha chaves. Os operadores aritméticos e lógicos são como em C ANSI; exceções (e.g., operadores lógicos e e ou) ocorreram em virtude do caráter didático que a pseudolinguagem proposta tem como objetivo.

As estruturas básicas de desvio e repetição seguem, em sua maioria, a estrutura da linguagem de programação C ANSI. Contudo, uma das estruturas que foi modificada com o intuito de apresentar um conceito de forma didática foi a estrutura de repetição para (for) que em PortuCol tem condições de parada fixas (menor ou igual, e maior ou igual). O objetivo dessa modificação foi de habituar o aluno a entender arranjos de tamanho $n$ como estruturas que são acessadas a partir da posição 0 até a posição $n-1$, o que acontece na maioria das linguagens de programação.

Usando o mesmo algoritmo descrito na Seção 2, os Algoritmos 3 e 4 apresentam dois exemplos do mesmo código em PortuCol e C ANSI, respectivamente.

Algoritmo 3. PortuCol

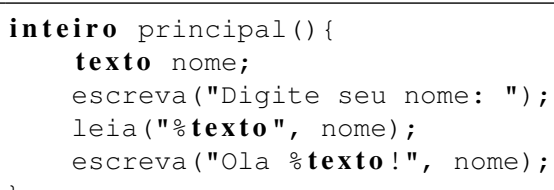

\subsection{Ferramenta PortuCo12C}

Para que os estudantes possam executar seus códigos escritos em PortuCol, foi criado um protótipo de uma ferramenta denominada PortuCo12C. Essa ferramenta realiza a tradução dos códigos em PortuCol para a linguagem C ANSI e, por conseguinte, já compila e gera o arquivo executável correspondente. Dessa forma, PortuCol2C permite que estudantes (i) "executem" seu código PortuCol e (ii) observem qual seria o código C ANSI correspondente, o que já os familiariza com linguagens de programação reais.

$\mathrm{Na}$ atual versão da ferramenta, a execução de PortuCo12C é por linha de comando, conforme pode ser observado na Figura 2. Dado um código em PortuCol (alg.pc), a execução ferramenta (portucol2c alg.pc) gera o código C ANSI correspondente (alg.c) e o arquivo executável (alg).

O tradutor foi implementado usando Lex e Yacc, ${ }^{1}$ que são ferramentas designadas para o desenvolvimento de compiladores e interpretadores [6]. O Lex realiza a análise léxica que consiste na leitura dos caracteres de entrada e produção de uma sequência de tokens, enquanto o Yacc executa a análise sintática que consiste na obtenção de uma cadeia

\footnotetext{
${ }^{1} \mathrm{http}: / /$ dinosaur.compilertools.net/
} 
Tabela 1. Equivalência de instruções PortuCol, C e Portugol

\begin{tabular}{|c|c|c|c|}
\hline Construções & PortuCol & C & Portugol \\
\hline Estrutura & $\begin{array}{l}\text { inteiro principal() }\{ \\
\}\end{array}$ & $\begin{array}{l}\text { int } \operatorname{main}() \\
\cdots\end{array}$ & $\begin{array}{l}\text { Algoritmo "nome" } \\
\text { var } \\
\text { inicio } \ldots \\
\text { fimalgoritmo }\end{array}$ \\
\hline Variável tipo inteiro & inteiro i; & $\begin{array}{l}\text { int } i ; \\
\text { short int } i ; \\
\text { long int } i ; \\
\text { long long int } i ;\end{array}$ & i: inteiro \\
\hline Variável tipo real & real d; & $\begin{array}{l}\text { float d; } \\
\text { double d; } \\
\text { long double d; }\end{array}$ & d: real \\
\hline Variável tipo lógico & $\operatorname{logico} b$; & bool b; (C99) & b: logico \\
\hline Variável tipo caractere & texto s; & char s; & s: caractere \\
\hline Variável tipo string & texto s; & char s[]; & s: caractere \\
\hline Variável tipo vetor & tipo arranjo[linhas]; & tipo arranjo[linhas]; & v: arranjo[linha1...linhaN] de tipo \\
\hline Variável tipo matriz & $\begin{array}{l}\text { tipo } \\
\text { arranjo[linhas] [colunas]; }\end{array}$ & $\begin{array}{l}\text { tipo } \\
\text { arranjo[linhas] [colunas]; }\end{array}$ & $\begin{array}{l}\text { v: arranjo[linhal.. linhaN, } \\
\text { colunal..colunaN] de tipo }\end{array}$ \\
\hline Ler valor & leia("施ipo", i); & scanf("tipo", i); & leia(i) \\
\hline Escrever & escreva ("..."); & printf("..."); & escreva ("...") \\
\hline Escrever com quebra de linha & escreva ("... $\backslash n ")$; & printf $(" \ldots \backslash n ")$; & escreval ("...") \\
\hline Escrever com parâmetros & $\begin{array}{l}\text { escreva ("... otipo ... } \\
\text { stipo...", a, b); }\end{array}$ & $\begin{array}{l}\text { printf("... \%tipo } \ldots \\
\text { otipo } \ldots \text {, a, b) }\end{array}$ & $\begin{array}{c}\text { escreval (".." , a, "...", } \\
b, " \ldots ")\end{array}$ \\
\hline Operação de atribuição & $a=b$ & $a=b$ & $\begin{array}{l}a<-b \\
a \quad:=b\end{array}$ \\
\hline Operação de adição & $a+b ;$ & $a+b ;$ & $a+b$ \\
\hline Operação de subtração & $a-b ;$ & $a-b ;$ & $a-b$ \\
\hline Operação de multiplicação & $a * b$ & $a * b ;$ & $a * b$ \\
\hline Operação de divisão & $\mathrm{a} / \mathrm{b} ;$ & $\mathrm{a} / \mathrm{b} ;$ & $a / b$ \\
\hline Operação de divisão inteira & $\mathrm{a} / \mathrm{b}$; & $\mathrm{a} / \mathrm{b}$; & $\mathrm{a} \operatorname{div} \mathrm{b}$ \\
\hline Operação de resto da divisão & $\mathrm{a} \div \mathrm{b}$; & $a \div b$ & $\begin{array}{l}a \div b \\
a \bmod b\end{array}$ \\
\hline Operação de potenciação & potencia $(a, b)$; & pow $(\mathrm{a}, \mathrm{b}) \quad<$ math. $\mathrm{h}>$ & $a \wedge b$ \\
\hline Operação de raiz quadrada & raizquadrada(a); & sqrt (a); $\quad<$ math.h> & RaizQ (a) \\
\hline Operação de concatenação & $\begin{array}{l}\text { concatena ("texto", "texto") ; } \\
\text { concatena ("texto", a); } \\
\text { concatena (a, "texto"); }\end{array}$ & $\begin{array}{l}\text { strcat ("texto", "texto"); } \\
\text { strcat ("texto", a); } \\
\text { strcat (a, "texto"); }\end{array}$ & $\begin{array}{l}\text { "texto" + "texto" } \\
\text { "texto" + a (string) } \\
\text { a (string) + "texto" }\end{array}$ \\
\hline Operação de tamanho do texto & tamanhotexto (a); & strlen(a); $\quad$ string.h> & compr (a) \\
\hline Operador menor & $\mathrm{a}<\mathrm{b}$ & $a<b$ & $a<b$ \\
\hline Operador menor ou igual & $a<=b$; & $\mathrm{a}<=\mathrm{b}$; & $a<=b$ \\
\hline Operador maior & $a>b$ & $a>b$ & $a>b$ \\
\hline Operador maior ou igual & $\mathrm{a}>=\mathrm{b}$ & $\mathrm{a}>=\mathrm{b}$; & $\mathrm{a}>=\mathrm{b}$ \\
\hline Operador igual & $\mathrm{a}==\mathrm{b}$ & $a==b$ & $a=b$ \\
\hline Operador diferente & $\mathrm{a}:=\mathrm{b}$ & $\mathrm{a}:=\mathrm{b}$; & $a<>b$ \\
\hline Operador e & $a$ e $b$ & $a \& \& b$ & $a$ e $b$ \\
\hline Operador ou & $\mathrm{a}$ ou $\mathrm{b}$ & $a \mid 1 b$ & $a$ ou b \\
\hline Operador de negação & !a & !a & nao a \\
\hline Condicional se & $\begin{array}{l}\text { se }(\text { condição }) \\
\text { s senà } \\
\text { \} }\end{array}$ & $\begin{array}{l}\text { if (condição) } \\
\text { \} else } \\
\text { \} } \quad \cdots\end{array}$ & $\begin{array}{l}\text { se condição entao } \\
\text { senao } \\
\text { fimse }\end{array}$ \\
\hline Condicional aninhado & $\begin{array}{l}\text { se (condição) }\{ \\
\text { \} senäo se (condição) }\{ \\
\text { \} senà } \\
\text { \} } \quad \text {... }\end{array}$ & $\begin{array}{l}\text { if (condição) }\{ \\
\text { \} el } \cdots \text { if (condição) \{ } \\
\text { \} el } \cdots \\
\text { \} } \quad \cdots\end{array}$ & $\begin{array}{l}\text { se condição entao } \\
\text { senao } \\
\text { se condicao entao } \\
\text { senao } \ldots \\
\text { fimse } \\
\text { fimse }\end{array}$ \\
\hline Condicional caso & $\begin{array}{l}\text { escolha (variável) } \\
\text { caso valor1: } \\
\quad \ldots \text {. } \\
\text { caso valor2, valor3: } \\
\text { padräo: } \\
\ldots\end{array}$ & $\begin{array}{c}\text { switch (variável) } \\
\text { case valor1: } \\
\text { break; } \\
\text { case valor2: } \\
\text { case valor3: } \\
\text { break; } \\
\text { default: } \\
\text { break; }\end{array}$ & $\begin{array}{l}\text { escolha variável } \\
\text { caso valor1 } \\
\text {... } \\
\text { caso valor2, valor } 3 \\
\text { outröàso } \\
\text { fimescoihà }\end{array}$ \\
\hline Condicional enquanto & $\begin{array}{l}\text { enquanto (condição) }\{ \\
\text { \} } \quad \cdots\end{array}$ & $\begin{array}{l}\text { while (condição) }\{ \\
\} \quad \cdots\end{array}$ & $\begin{array}{l}\text { enquanto condição faca } \\
\text { Fimenquanto }\end{array}$ \\
\hline Condicional para & $\begin{array}{l}\text { para }(\operatorname{variável}=\text { valor } 1 ; \\
\operatorname{valorN} ; \mathrm{P}) \text { i } \\
\quad \ldots\end{array}$ & $\begin{array}{l}\text { for (variável = valorl; } \\
\text { variável <= valorn; } \\
\text { variável += P) \{ } \\
\quad \cdots\end{array}$ & $\begin{array}{l}\text { para variável de valor } 1 \\
\text { ate valorn passo } \mathrm{P} \text { faca } \\
\ldots \ldots \\
\text { fimpara }\end{array}$ \\
\hline Comando de interrupção & interrompa; & break; & interrompa \\
\hline Criar procedimento & $\begin{array}{l}\text { semretorno nome (tipo } \\
\text { parâmetro, } \ldots . \text { ) } \\
\quad \cdots\end{array}$ & $\begin{array}{l}\text { void nome (tipo parâmetro, } \\
\cdots \cdots)\} \\
\text { \} } \quad \cdots\end{array}$ & $\begin{array}{l}\text { procedimento nome } \\
\text { parâmetro: tipo; } . . .) \\
\text { var } \ldots \\
\text { inicio } \\
\text { fimpröedimento }\end{array}$ \\
\hline Criar função & $\begin{array}{l}\text { tipo nome (tipo parâmetro, } \\
\cdots \cdots) \text { \{ } \\
\text { retorne valor; } \\
\text { \} }\end{array}$ & 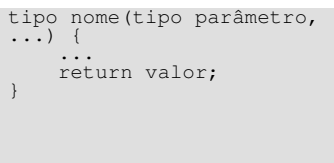 & $\begin{array}{l}\text { funcao nome (parâmetro: } \\
\text { tipo; } \ldots \text { ): tipo } \\
\text { var } \\
\text { inicio } \\
\text { retorne valor } \\
\text { fimfuncao }\end{array}$ \\
\hline
\end{tabular}




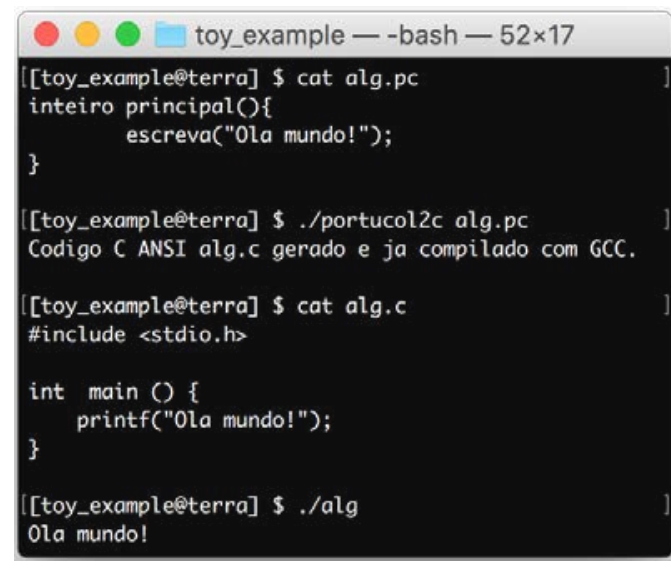

Figura 2. Execução do PortuCol2C

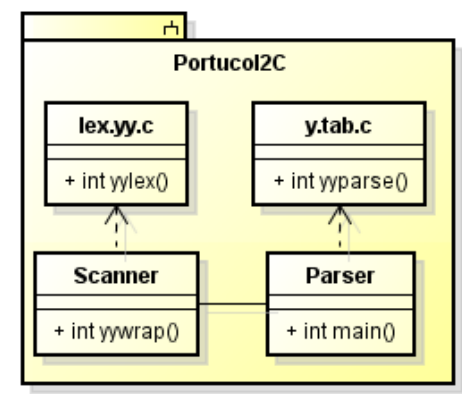

Figura 3. Arquitetura do PortuCo12C

de tokens e verificação de quaisquer erros de sintaxe de uma forma inteligível [1]. Conforme ilustrado na Figura 3, a ferramenta segue então uma arquitetura com os seguintes dois módulos principais:

1. Scanner: Responsável por realizar a análise léxica do código-fonte escrito em PortuCol. Sua implementação consiste em definições de expressões regulares a serem convertidas em tokens tais como números, variáveis e palavras reservadas. Posteriormente, são definidas as regras para cada token, sendo que no caso do Scanner as regras estabelecidas consistem em retornar cada token para o Parser. A compilação do Scanner pelo Lex gera o arquivo lex.yy.c, o qual é utilizado para viabilizar a detecção dos tokens no código-fonte do PortuCol.

2. Parser: Responsável por realizar a análise sintática do código-fonte escrito em PortuCol. O Parser é constituído por regras formadas através dos tokens recebidos pelo Scanner, sendo que cada regra possui subrotinas associadas. No nosso caso, as subrotinas têm como finalidade imprimir, no arquivo de saída, a tradução para código C ANSI da sequência de tokens relacionado à cada regra. A compilação do Parser pelo Yacc gera o arquivo y.tab.c, o qual é utilizado para possibilitar a análise sintática dos tokens.

\section{Avaliação}

\subsection{Questões de Pesquisa}

Um estudo foi projetado para responder às seguintes questões de pesquisa:

QP \#1 - PortuCol se aproxima à C ANSI mais do que Portugol?

QP \#2 - PortuCol se aproxima à C ANSI mais do que Portugol se aproxima à Pascal?

\subsection{Dataset $^{2}$}

Para efetivamente comparar a similaridade entre algoritmos nas linguagens PortuCol, C, Portugol e Pascal, foram especificados 30 algoritmos, os quais foram implementados em cada uma das linguagens. A Tabela 3 descreve sucintamente os algoritmos implementados e aponta as principais estruturas utilizadas.

\footnotetext{
${ }^{2}$ Dataset publicamente disponível em: http://www.dcc.ufla.br/ terra/papers/2016_wei_portucol
} 
Tabela 3. Descrição dos algoritmos

\begin{tabular}{|c|c|c|}
\hline Algoritmo & Descrição & Principais estruturas utilizadas \\
\hline Alg_1 & Cadastro e acesso de produtos & Procedimentos, enquanto, para, caso, se \\
\hline Alg_2 & Equação quadrática & Tipo de dado real, se senao se \\
\hline Alg_3 & Idade por ano de nascimento & Tipo de dado arranjo, para \\
\hline Alg_4 & Bubble sort & Tipo de dado arranjo, para, se \\
\hline Alg_5 & Busca binária & Função, enquanto, se senao se \\
\hline Alg_6 & Busca em arranjo & Faca enquanto, se, senao \\
\hline Alg_7 & Calculadora $(+,-, *)$ & Caso, tipo de dado texto \\
\hline Alg_8 & Média de notas & Leitura de entrada, se, senao \\
\hline Alg_9 & Soma e subtração de reais & Para, se, senao \\
\hline Alg_10 & Combinação & Escrita e leitura de dados, Para \\
\hline Alg_11 & Decomposição de inteiro & Para, faca enquanto, se \\
\hline Alg_12 & Aprovação de aluno por nota & Caso \\
\hline Alg_13 & Fatorial recursivo & Função recursiva, se senao se \\
\hline Alg_14 & Fibonacci iterativo & Função, para \\
\hline Alg_15 & Selection sort & Para, tipo de dado arranjo, se \\
\hline Alg_16 & Mostrar coluna de matriz & Matriz 4x4, para \\
\hline Alg_17 & Média de reais & Função, tipo de dados real \\
\hline Alg_18 & Inverter texto & Tipo de dados texto, para \\
\hline Alg_19 & Números palíndromos & Enquanto, se, senao \\
\hline Alg_20 & Primos até certo número & Para, enquanto, se \\
\hline Alg_21 & Aproximação de $\mathrm{Pi}$ & Para, operação de raiz quadrada \\
\hline Alg_22 & Potenciação de inteiros (x^y) & Para \\
\hline Alg_23 & Comb sort & Enquanto, se, para \\
\hline Alg_24 & Soma de matrizes & Matrizes $2 \times 2$, para \\
\hline Alg_25 & Soma de algarismos & Enquanto, operação de resto de divisão \\
\hline Alg_26 & Classificação de texto por caso & Caso, comparação de texto \\
\hline Alg_27 & Validação de CPF & Para, se, conversão de texto para inteiro \\
\hline Alg_28 & Divisão de arranjos entre par e ímpar & Repita enquanto, para, se, senao \\
\hline Alg_29 & Aproximação de raiz quadrada & Se, enquanto, senao \\
\hline Alg_30 & Concatenação e comparação de texto & Se, senao, operações de concatenação e comparação de texto \\
\hline
\end{tabular}

\subsection{Similaridade Textual}

Para obter a similaridade entre os algoritmos implementados em PortuCol, C, Portugol e Pascal foi utilizada a similaridade de cosseno (cosine similarity) - técnica mais adotada em recuperação de informação e mineração de texto para comparação de documentos [7] a qual mensura a similaridade de dois documentos com base no ângulo entre seus vetores representativos. ${ }^{3}$

Portanto, a similaridade de cosseno entre dois documentos $d_{1}$ e $d_{2}$ é computada a partir dos seus vetores representativos $\vec{V}\left(d_{1}\right)$ e $\vec{V}\left(d_{2}\right)$ conforme Equação 1 .

$$
\operatorname{sim}\left(d_{1}, d_{2}\right)=\frac{\vec{V}\left(d_{1}\right) \cdot \vec{V}\left(d_{2}\right)}{\left|\vec{V}\left(d_{1}\right)\right|\left|\vec{V}\left(d_{1}\right)\right|}
$$

onde $\vec{V}\left(d_{i}\right)$ denota o vetor derivado de um documento $d_{i}$, com um componente no vetor para cada termo do dicionário. Assim, cada termo do documento é armazenado uma única vez no vetor e é associado a um peso. O peso dos termos normalmente leva em consideração o $t f-i d f_{t, d}$, onde $t f$ é a frequência dos termos (Term Frequency), $i d f$ é a frequência inversa do documento (Inverse Document Frequency), $t$ representa os termos e $d$ o documento. ${ }^{4}$ Enfim, a similaridade entre dois documentos consiste na razão entre (i) $o$ produto escalar e (ii) o produto das normas euclidianas dos dois vetores representativos.

\footnotetext{
${ }^{3}$ A similaridade varia de 0 (nada similares) a 1 (totalmente similares).

${ }^{4}$ Particularmente neste artigo, foi considerada apenas a frequência dos termos, uma vez que diminuir o peso de termos frequentes não é desejável.
} 


\subsection{Metodologia}

Foram conduzidas as seguintes atividades para se responder às questões de pesquisa:

1. Definir uma particular versão de Portugol, devido à existência de variações do Portugol encontradas na literatura, onde diferentes ferramentas $[3,5,9,8,4]$ possuem sua próprias variações de Portugol. Assim, foi escolhida a variante adotada pelo VisuAlg - um interpretador de Portugol desenvolvido por Souza [4] - a qual é completa e amplamente aceita.

2. Construir um dataset contendo uma vasta gama de algoritmos implementados nas linguagens PortuCol, C, Portugol e Pascal (veja Seção 4.2).

3. Comparar a similaridade textual - utilizando cosine similarity descrito na Seção 4.3 - dos algoritmos implementados na linguagem PortuCol com C, Portugol com $\mathrm{C}$ e Portugol com Pascal. É importante mencionar que textos referentes à inclusão de bibliotecas (e.g. \#include $<$ stdio.h $>$ e uses crt) foram retirados dos códigos $\mathrm{C}$ e Pascal.

4. Determinar estatisticamente a relação de similaridade de PortuCol com $\mathrm{C}$ e de Portugol com C, a fim de responder a QP \#1.

5. Determinar estatisticamente a relação de similaridade de PortuCol com $\mathrm{C}$ e de Portugol com Pascal, a fim de responder a QP \#2.

\subsection{Resultados}

A Tabela 4 reporta os resultados da similaridade textual dos algoritmos implementados na linguagem Portugol com C (coluna 1), PortuCol com C (coluna 2) e Portugol com Pascal (coluna 3). A Figura 4 representa visualmente o intervalo de confiança para a média das similaridades textuais de cada par de linguagens.

Tabela 4. Similaridade Textual

\begin{tabular}{|c|c|c|c|}
\hline Algoritmo & Portugol/C & PortuCol/C & Portugol/Pascal \\
\hline Alg_1 & 0.4004 & 0.6031 & 0.4365 \\
\hline Alg_2 & 0.7009 & 0.6824 & 0.7607 \\
\hline Alg_3 & 0.6348 & 0.7314 & 0.7291 \\
\hline $\mathrm{Alg}_{4}$ & 0.7824 & 0.8713 & 0.7850 \\
\hline Alg_5 & 0.6803 & 0.7712 & 0.6591 \\
\hline Alg_6 & 0.5866 & 0.7072 & 0.6256 \\
\hline $\mathrm{Alg} 7$ & 0.6674 & 0.6292 & 0.7227 \\
\hline Alg_8 & 0.6190 & 0.5930 & 0.6347 \\
\hline Alg_9 & 0.3036 & 0.7593 & 0.6543 \\
\hline Alg_10 & 0.7386 & 0.6616 & 0.8338 \\
\hline Alg_11 & 0.7262 & 0.8586 & 0.7523 \\
\hline Alg_12 & 0.2761 & 0.3639 & 0.5355 \\
\hline Alg_13 & 0.6385 & 0.6468 & 0.6156 \\
\hline Alg_14 & 0.6230 & 0.6863 & 0.5823 \\
\hline Alg_15 & 0.7190 & 0.8915 & 0.7118 \\
\hline Alg_16 & 0.5420 & 0.6057 & 0.5809 \\
\hline Alg 17 & 0.4449 & 0.4355 & 0.5536 \\
\hline Alg_18 & 0.6502 & 0.7662 & 0.7963 \\
\hline Alg_19 & 0.8511 & 0.8685 & 0.8348 \\
\hline Alg_20 & 0.3073 & 0.7611 & 0.7110 \\
\hline Alg_21 & 0.3267 & 0.7708 & 0.7780 \\
\hline Alg_22 & 0.6261 & 0.6199 & 0.6581 \\
\hline Alg_23 & 0.8580 & 0.9192 & 0.8247 \\
\hline Alg_24 & 0.0407 & 0.8187 & 0.9045 \\
\hline Alg_25 & 0.1079 & 0.8729 & 0.7621 \\
\hline Alg_26 & 0.6490 & 0.7286 & 0.7338 \\
\hline Alg_27 & 0.6202 & 0.8472 & 0.5964 \\
\hline Alg_28 & 0.5844 & 0.6647 & 0.6306 \\
\hline Alg_29 & 0.8454 & 0.8956 & 0.8604 \\
\hline Alg_30 & 0.4611 & 0.5484 & 0.4665 \\
\hline Média $(\bar{x})$ & 0.5671 & 0.7193 & 0,6910 \\
\hline Dev. Pad. $(s)$ & 0.2089 & 0.1361 & 0,1161 \\
\hline
\end{tabular}

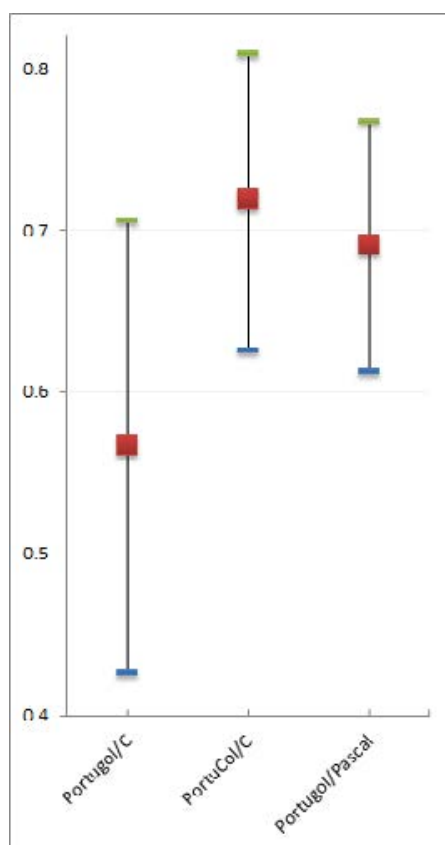

Figura 4. Intervalo de confiança 


\section{QP \#1 - PortuCol se aproxima à C ANSI mais do que Portugol?}

Foram comparadas as similaridades textuais dos algoritmos implementados (i) em Portugol e em $\mathrm{C}$ e (ii) em PortuCol e em $\mathrm{C}$, cujos resultados podem ser observados nas primeiras duas colunas da Tabela 4 e nas duas primeiras linhas da Figura 4.

O dataset disponível para avaliação apresenta os mesmos algoritmos implementados em diferentes linguagens (veja Seção 4.2). Isso permite que seja conduzida uma observação pareada dos resultados de similaridade textual. Portanto, considerando uma distribuição normal dos resultados, a distribuição probabilística $t$-Student foi utilizada no cálculo do intervalo de confiança para responder à QP \#1, conforme Equação 2.

$$
I C=\bar{x} \pm t_{\left[1-\frac{\alpha}{2} ; n-1\right]}\left(\frac{s}{\sqrt{n}}\right)=0,1523 \pm 3,6590\left(\frac{0,2158}{\sqrt{30}}\right)=[0,0081,0,2964]
$$

O dataset utilizado possui trinta algoritmos $(n=30)$. A diferença da similaridade textual entre cada algoritmo Portugol/C e PortuCol/C (colunas 1 e 2 da Tabela 4) foi calculada. Assim, obteve-se a média aritmética $(\bar{x}=0,1523)$ e o desvio padrão $(s=0,2158)$ das diferenças. A confiança adotada para as amostras da distribuição de $t$-Student foi $99,9 \%$ com grau de liberdade de 29 , onde $t_{[0.9995 ; 29]}$ é igual a 3,659. Assim, o teste $t$ resultou no intervalo de $[0,0081,0,2964]$, o qual não inclui zero. Isso indica que, com $99,9 \%$ de confiança, PortuCol se aproxima $26,85 \%$ mais à C ANSI do que Portugol.

QP \#2 - PortuCol se aproxima à C ANSI mais do que Portugol se aproxima à Pascal?

Similarmente à QP \#1, foram comparadas as similaridades textuais dos algoritmos implementados (i) em PortuCol e em C e (ii) em Portugol e em Pascal, cujos resultados podem ser observados nas últimas duas colunas da Tabela 4 e nas duas últimas linhas da Figura 4. É importante mencionar que os resultados comparativos entre PortuCol e $\mathrm{C}$ já tinham sido previamente calculados na resposta à QP \#1 .

Considerando uma distribuição normal dos resultados, a distribuição probabilística $t$-Student novamente foi utilizada no cálculo do intervalo de confiança para responder à QP \#2, conforme Equação 3.

$$
I C=\bar{x} \pm t_{\left[1-\frac{\alpha}{2} ; n-1\right]}\left(\frac{s}{\sqrt{n}}\right)=0,0283 \pm 3.659\left(\frac{0.1006}{\sqrt{30}}\right)=[-0,0389,0,0955]
$$

Similarmente à $Q P \# 1$, a confiança adotada para as amostras da distribuição de $t$-Student foi $99,9 \%$ com grau de liberdade de 29. Assim, o teste $t$ resultou no intervalo de [-0,0389, 0,0955]. Como o intervalo de confiança inclui zero, podemos considerar que o PortuCol se aproxima à C ANSI da mesma maneira que Portugol se aproxima à Pascal.

\section{Considerações Finais}

O uso de pseudolinguagens no ensino de Lógica de Programação e Algoritmos é uma estratégia muito utilizada em IES. Embora C ANSI e Java sejam as linguagens de programação mais utilizadas no desenvolvimento de sistemas de software [10], a pseudolinguagem mais utilizada no Brasil é o Portugol $[9,11]$, que é inspirada no obsoleto Pascal.

\footnotetext{
${ }^{5} \mathrm{O}$ teste $t$ resulta em um intervalo de confiança. Caso o intervalo inclua zero, as amostras não são estatisticamente diferentes. Caso contrário, pode-se afirmar que as amostras são estatisticamente diferentes, o que, neste caso, indica que a similaridade em um par de linguagens é maior que na outra.
} 
Diante desse cenário, este artigo propôs PortuCol, uma pseudolinguagem de programação com instruções em português assim como Portugol, porém inspirada em C ANSI. Como contribuição acadêmica, uma análise estatística da similaridade textual demostrou que: (i) a pseudolinguagem PortuCol é estatisticamente 26,85\% mais similar à C ANSI do que Portugol, o que proporciona uma transição mais natural ao estudante ao aprender linguagens do estado-da-prática como C ANSI e Java; e (ii) PortuCol é para C ANSI o que Portugol é para Pascal, o que retrata apenas uma adaptação da pseudolinguagem às linguagens do estado-da-prática. Como contribuição prática, este artigo também apresenta PortuCo12C, uma ferramenta que executa código PortuCol através da prévia tradução para código C ANSI, o que possibilita aos estudantes se familiarizem com a linguagem C ANSI através da observação do seu código PortuCol traduzido.

$\mathrm{O}$ dataset dos algoritmos utilizados na avaliação e a ferramenta PortuCol2C (binário e código fonte) estão publicamente disponíveis em: http://www.dcc.ufla.br/ terra/papers/2016_wei_portucol

Como trabalhos futuros, pretende-se (i) avaliar o uso da pseudolinguagem PortuCol no ensino de Lógica de Programação e Algoritmos e (ii) melhorar a ferramenta PortuCo12C com um ambiente de desenvolvimento integrado (IDE) e um depurador.

\section{Agradecimentos}

Este trabalho foi apoiado pela FAPEMIG, CAPES e CNPq.

\section{Referências}

[1] Alfred V. Aho, Ravi Sethi, and Jeffrey D. Ullman. Compiladores: Principios, técnicas e ferramentas. LTC, 2 edition, 2008.

[2] Ricardo Luiz B. L. Campos. Lógica de programação: Há como melhorar o aprendizado fugindo dos padrões estabelecidos nos livros didáticos e adotados pela maioria dos docentes? In XVII Congresso Iberoamericano de Educación Superior em Computación (CIESC), pages 22-25, 2009.

[3] Rafael de Santiago and Rudimar Luís Scaranto Dazzi. Ferramenta de apoio ao ensino de algoritmos. In XIII Seminário de Computação (SEMINCO), pages 1-8, 2004.

[4] Cláudio Morgado de Souza. VisuAlg - ferramenta de apoio ao ensino de programação. TECCEN, 2(2):1-9, 2009.

[5] Mauri Ferrandin and Simone Lilian Stephani. Ferramenta para o ensino de programação via internet. In I Congresso Sul Catarinense de Computação (SULCOMP), pages 1-8, 2005.

[6] John R. Levine, Tony Mason, and Doug Brown. Lex \& Yacc. O’Reilly, 2 edition, 1992.

[7] Christopher D. Manning, Prabhakar Raghavan, and Hinrich Schütze. Introduction to information retrieval. Cambridge University Press, 2008.

[8] António Manso, Luís Oliveira, and Célio Gonçalo Marques. Ambiente de aprendizagem de algoritmos Portugol IDE. In VI Conferência Internacional de TIC na Educação, pages 969-983, 2009.

[9] Luiz F. Noschang, Fillipi Pelz, Elieser A. de Jesus, and André L. A. Raabe. Portugol Studio: Uma IDE para iniciantes em programação. In XXII Workshop sobre Educação em Computação (WEI), pages 1287-1296, 2014.

[10] TIOBE. Programming languages index. http://www.tiobe.com/tiobe_index, March 2016.

[11] Adriana Salvador Zanini and André Luís Alice Raabe. Análise dos enunciados utilizados nos problemas de programação introdutória em cursos de ciência da computação no Brasil. In XX Workshop sobre Educação em Computação (WEI), 2012. 